\title{
An Iterative Technique for the Synthesis of Active Antenna Oscillator Arrays
}

\author{
Theodoros N. Kaifas and John N. Sahalos \\ Radiocommunications Laboratory, Aristotle University of Thessaloniki, 54124 Thessaloniki, Greece \\ Correspondence should be addressed to John N. Sahalos, sahalos@auth.gr
}

Received 1 September 2008; Revised 16 December 2008; Accepted 5 February 2009

Recommended by Stefano Selleri

\begin{abstract}
A design procedure for the synthesis of a coupled active antenna oscillator array is presented. Such an array is synthesized by deriving two sets of parameters: the radiators' positions and the oscillators' outputs. The outputs are used to excite the radiators. Minimization of the mean square error between the desired pattern and the resulting one is made. Synthesis starts from an initial array, which is perturbed iteratively by varying simultaneously the element excitations and positions. In the iteration, the first variation of the cost function is set equal to zero. The final array results from the last iteration, where the stopping criteria are met. The procedure designs simultaneously both the antenna and the attached coupled oscillator array providing viable solutions. The second by properly configuring the tuning parameters through the use of closed-form formulas. The resulting arrays are shown to comply with the desired pattern and the nonlinear dynamics thus proving the validity of our method.
\end{abstract}

Copyright (c) 2009 T. N. Kaifas and J. N. Sahalos. This is an open access article distributed under the Creative Commons Attribution License, which permits unrestricted use, distribution, and reproduction in any medium, provided the original work is properly cited.

\section{Introduction}

It is well known that the angular distribution of the radiated power from an antenna array depends on the geometry and the excitation of the elements [1]. In a coupled active antenna oscillator linear array consisting of identical and parallel elements, the pattern is expressed in the following form:

$$
\begin{gathered}
F(\theta)=\sum_{k=1}^{N}\left(\tilde{V}_{k}\right) f_{k}(\theta) e^{j(2 \pi / \lambda) z_{k} \cos (\theta)} \\
V_{k}=\left|\tilde{V}_{k}\right| \\
\phi_{k}=\angle \tilde{V}_{k},
\end{gathered}
$$

where the excitation $\tilde{V}_{k}$ is the complex voltage of the oscillator output, $\left(V_{k}=\left|\tilde{V}_{k}\right|\right.$ amplitude and $\phi_{k}=\angle \tilde{V}_{k}$ the respective phase), $f_{k}(\theta)$ is the pattern, and $z_{k}$ is the abscissa of the $k$ th element, respectively. $\lambda$ is the free-space wavelength at the operating frequency.

The two key sets of parameters that are important in the design are the boxed terms of (1). The antenna array synthesis mainly focuses on the excitation [2-4], while efforts aiming at extracting the positions of the array elements are scarce [5]. In our present study, we cope either with one of the above or with both sets simultaneously. A perturbation method that provides iteratively the excitation and the placement of the elements in a quest of matching the resulting far-field with the desired one is applied. The whole synthesis is formulated as an optimization problem over a vector containing the excitation and the element positions. The cost function is the mean square error between the desired and the attained pattern.

Apart from attaining the necessary parameters of the antenna array, it is of equal importance the problem of feeding the array by a proper network. In the literature, one can find several approaches to the feeding networks [1], containing corporate feeds and feeding matrices [6]. Apart from passive networks, there are others that utilize active circuitry. Among them, the active feeding networks consisting of coupled oscillator arrays [7] are of specific interest. It has been found that such devices allow for the manipulation of the phase distribution without additional phase-shifting circuitry, suggesting a potential for low-cost beam-scanning systems. In the past, most of the studies on coupled oscillator arrays analyze the problem of beam steering [8-10] and of null control of the pattern, [11-13]. In 


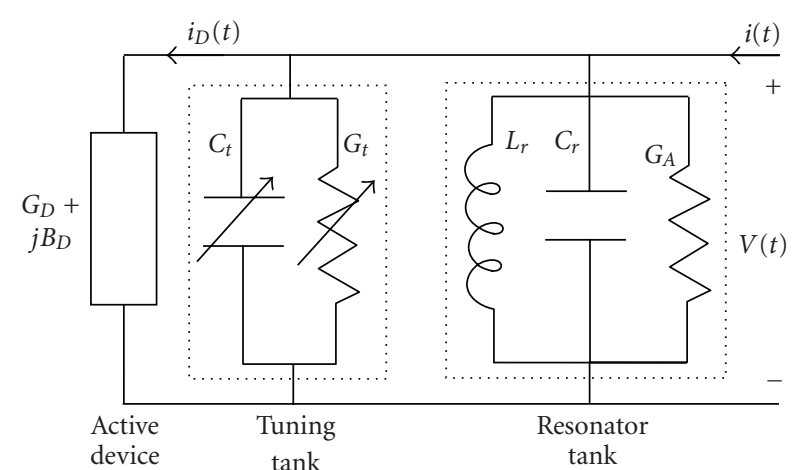

FIGURE 1: The equivalent circuit of the oscillator in the current-sum form.

the current work, we extend the functionality of the coupled oscillator arrays to more general pattern synthesis problems.

It is noticed that there are cases where a uniform excitation of a linear phased array and a nonuniform placement is enough to solve the synthesis problem. In this case, constraining the oscillators' output voltage to a common value reduces the nonlinear dynamics of the system. Alternatively, one can keep the phases equal and seek a design for different amplitudes. The required excitation distribution for all the simplifying cases, and for the general one (see the design examples), can be readily implemented with proper tune of control parameters, such as the varactor capacitance and the PIN conductance of the oscillators. The relative closed-form formulas are presented for the case of the mutually synchronized coupled oscillator array.

The paper is organized as follows. In Section 2, the coupled oscillator array (COA) theory is reviewed, and the key aspects related to the present study are underlined. The system that contains both, the antenna and the oscillators, is presented in Section 3, where closed-form formulas are derived to design the mutually synchronized oscillator array. The formulation and the solution of the radiation pattern shaping optimization problem for the above active antenna array are given in Section 4. In Section 5, design examples that verify the effectiveness of the method follow. Finally, some concluding remarks are given in Section 6.

\section{The Coupled Oscillator Array}

2.1. Single Oscillator Description. A model that has been widely used for microwave oscillator circuits is the canonical oscillator model in a parallel current-sum form which leads to the Van der Pol equation for certain nonlinearities [14, 15]. The equivalent circuit of the oscillator can be extracted from measurements [16] or from simulation [17], and is given in Figure 1.

The oscillator is assembled by three distinct parts: the active device, the tuning tank, and the resonator tank. The active device provides the nonlinear voltage dependent negative conductance. The tuning tank is responsible for the adjustment of the tuning parameters in order to feed the attached antenna element with the proper excitation. Last, the resonator tank includes the parallel resonance circuit of the oscillator and the antenna elements.

In detail, the active device is represented as a negative conductance as follows:

$$
G_{D}(|V|)=-a+b|V|^{2},
$$

where $|V|$ is the amplitude of the output voltage, and $a, b$ are constants characterizing the nonlinear device.

The voltage-dependent susceptance of the nonlinear device is denoted by $B_{D}(|V|)$. Usually, the susceptance is independent on the device characteristics and can be neglected. In this case, which is the one that we follow, the current of the nonlinear device obeys

$$
i_{D}(t)=Y \cdot V(t)=G_{D} \cdot V(t)=\left(-a+b \cdot V^{2}(t)\right) \cdot V(t) .
$$

The reactive energy of the circuit is stored into the $L C$ parallel circuit giving a free-running circular frequency of

$$
\omega_{o, k}=\frac{1}{\sqrt{L_{r} C_{o, k}}}=\frac{1}{\sqrt{L_{r}\left(C_{r}+C_{o, t}\right)}}
$$

for the $k$ th oscillator.

In our case, the capacitance $C_{t}$ would be a varactor that is controlled by a voltage. This voltage will be the tuning parameter which for the $k$ th oscillator is $V_{k}^{t}$. The varactor capacitance follows the well-known equation [18]:

$$
C_{t, k}=\frac{2 C_{o, t}}{\left(1+V_{k}^{t} / V_{o}\right)^{\Gamma}},
$$

where $V_{o}=1.2 \mathrm{~V}$ (for a GaAs device), $C_{o, t}=1 \mathrm{pF}$ is the capacitance for $V_{o}$ tuning voltage, and $\Gamma$ is the varactor exponent (usually taken equal to unity, a choice also followed here). Alternatively, other types of voltage controlled capacitors $[19,20]$ or even voltage controlled inductors [21] can be used instead.

The conductance $G_{t}$ represents additional losses in the oscillator circuit. This would be the second (together with the $\left.C_{t, k}\left(V_{k}^{t}\right)\right)$ tuning parameter. In our case, the conductance $G_{t}$ would be a PIN diode variable resistor controlled by its forward current $I_{k}^{t}$. The PIN conductance follows the wellknown equation $[22,23]$ :

$$
G_{t, k}=\frac{v_{o}}{I_{k}^{t}}
$$

Here, we use $v_{o}=20 \mathrm{mV}$. Alternatively, other types of variable resistors can be used instead [24].

To find the total conductance, one should add to $G_{t}$ the input conductance $G_{A}$ of the radiating element of the array.

Let us now compute the total admittance $Y$ of the circuit:

$$
\begin{aligned}
& i(t)=(\underbrace{Y_{D}}_{=-G_{D}}+\underbrace{Y_{C}}_{=j\left(C_{t}+C_{r}\right) \omega}+\underbrace{Y_{L}}_{=1 / j L_{r} \omega}+Y_{G_{t}}+Y_{G_{A}}) V(t) \\
& \Longrightarrow Y=-\left(G_{D}(|V|)-G_{t}-G_{A}\right)+j\left(C_{t}+C_{r}\right) \frac{\omega^{2}-\omega_{o}^{2}}{\omega} .
\end{aligned}
$$


We assume that the resonant circuit of the oscillator is of high $Q$. Thus, the output voltage follows, nearly, a sinusoidal waveform with the following phasor representation:

$$
\begin{aligned}
V(t) & =V_{0}(t) \cos (\omega t+\phi(t)) \\
& =\operatorname{Re}\left\{V_{0}(t) e^{j \phi(t)} e^{j \omega t}\right\} \\
& =\operatorname{Re}\left\{\tilde{V}(t) \cdot e^{j \omega t}\right\} .
\end{aligned}
$$

2.2. Coupled Oscillator Array Description. To complete the analysis, we suppose (see Figure 2) that we have $N$ oscillators which are joined together using a linear coupling network [25].

The governing equation reads

$$
Y_{i} \cdot \tilde{V}_{i}+\sum_{j=1}^{N} Y_{i j} \cdot \tilde{V}_{j}=0,
$$

where $Y_{i}$ is given by (7), and $\tilde{V}_{i}$ is defined in (8) as the complex output voltage of the $i$ th oscillator. Also,

$$
Y_{i j}=G_{i j}+j B_{i j}
$$

is the mutual admittance between the output nodes of the $i$ th and jth oscillators. Using the above, it can be shown [16-27] for the voltage amplitude $V_{k}$ of the $k$ th oscillator that

$$
\begin{aligned}
\frac{d V_{k}}{d t} & +\frac{\omega_{o, k}}{2 Q_{k}} V_{k}\left[1-\frac{\left(-a_{k}+b_{k} V_{k}^{2}\right)}{G_{t, k}+G_{A}}\right] \\
& =\frac{1}{2 C_{k}}\left(\sum_{l=1}^{N} V_{l}\left[G_{l k} \cos \left(\phi_{l}-\phi_{k}\right)-B_{l k} \sin \left(\phi_{l}-\phi_{k}\right)\right]\right),
\end{aligned}
$$

where

$$
Q_{k}=\frac{\omega_{o, k} C_{k}}{\left(G_{t, k}+G_{A}\right)},
$$

with $C_{k}=C_{t, k}+C_{r}$.

Often the following substitution is made:

$$
\begin{gathered}
1-\frac{\left(-a_{k}+b_{k} V_{k}^{2}\right)}{G_{t, k}+G_{A}} \approx \mu_{k}\left(a_{o, k}^{2}-\left|V_{k}\right|^{2}\right), \\
a_{o, k}=\sqrt{\frac{a_{k}+G_{t, k}+G_{A}}{b_{k}}}, \\
\mu_{k}=\frac{b_{k}}{G_{t, k}+G_{A}},
\end{gathered}
$$

where $\mu_{k}$ is a parameter that characterizes the oscillator, while $\alpha_{o}$ is the free-running amplitude of the output voltage. This weak quadratic nonlinearity is the simplest nonlinearity that approximates the behavior of a wide range of active devices.

Apart from the time evolution of the voltage amplitudes given in (11) for the phase, we have

$$
\begin{aligned}
\frac{d \phi_{k}}{d t}= & -\omega+\omega_{o, k}+\frac{1}{2 V_{k} C_{k}} \\
& \times\left(\sum_{l=1}^{N} V_{l}\left[G_{l k} \sin \left(\phi_{l}-\phi_{k}\right)+B_{l k} \cos \left(\phi_{l}-\phi_{k}\right)\right]\right) .
\end{aligned}
$$

Equation (11) in conjunction with (14) fully describes the coupled oscillator array. The above should provide a framework for the design of an antenna oscillator array.

\section{Design of the Mutually Synchronized Array}

In Figure 3 [28], an antenna array with the corresponding oscillators is presented. In this mutually synchronized array, each oscillator is bilaterally coupled to the neighboring array elements. The configuration of Figure 3was first proposed by Stephan $[8,15]$ and named as "interinjection-locked" oscillators. Mutual coherence is achieved via the injectionlocking process. The steady-state phase relationships of each oscillator depend on its neighbors' amplitude and phase. A nonlinear system of simultaneous equations must be solved to determine the self-consistent amplitude and phase relationships [26]. In such systems, (11) and (14) take the form

$$
\begin{aligned}
\frac{d V_{k}}{d t}= & \frac{V_{k}}{2 C_{k}}\left[G_{t, k}+G_{A}+a_{k}-b_{k} V_{k}^{2}\right] \\
& +\frac{1}{2 C_{k}} \sum_{l=1}^{N}\left[V_{l} \kappa_{l k} \cdot \cos \left(\phi_{l}-\phi_{k}+\Phi_{l k}\right)\right] \\
\frac{d \phi_{k}}{d t}= & -\omega+\omega_{o, k}+\frac{1}{2 C_{k}} \sum_{l=1}^{N}\left[\frac{V_{l}}{V_{k}} \kappa_{l k} \cdot \sin \left(\phi_{l}-\phi_{k}+\Phi_{l k}\right)\right],
\end{aligned}
$$

where $\kappa_{l k}$ are different from zero only when $|l-k| \leq 1$ since only the nearest neighbor coupling is assumed. There are various sets of parameters in (15). Those are the oscillator detuning, $\left(\omega-\omega_{o, k}\right)$, the un-normalized coupling strengths, $\left(\kappa_{l k}=\left|G_{l k}+j B_{l k}\right|\right)$, and the coupling phases, $\left(\Phi_{l k}=\angle\left(G_{l k}+\right.\right.$ $\left.\left.j B_{l k}\right)\right)$. There are also the capacitance and the conductance of each one of the oscillators. Since our aim is to manipulate the amplitude and the phase distribution of the active antenna array, any set of parameters could be used for control. Here, we eliminate the coupling phases and we use the conductance of the PIN diodes and the capacitance of the varactors to control the steady state of the amplitudes and phases.

Before we present the followed procedure, some issues relative to the coupling network should be addressed. To couple the oscillators, we use resistively loaded lines [29], whose characteristic impedance $Z_{0}$ and phase $\Phi$ of the electrical length are

$$
Z_{o}=R, \quad \Phi=2 \pi .
$$

For such lines, it is

$$
Y_{i j}= \begin{cases}\frac{n_{i}}{2 R}, & i=j, \\ -\frac{1}{2 R}, & |i-j|=1, \\ 0, & \text { otherwise, }\end{cases}
$$

where $n_{i}=2$ except for the edge elements where it is equal to one.

Using this coupling network, closed-form expressions for the tuning parameters can be derived. So, for $Y_{i j}$ given in 
(17), (15), for the steady state, where the time derivatives vanish, reads

$$
\begin{aligned}
& V_{k}\left[G_{t, k}+G_{A}+a_{k}-b_{k} V_{k}^{2}\right] \\
& =\frac{-1}{2 R}\left\{\begin{array}{cl}
V_{1}-V_{2} \cos \left(\phi_{2}-\phi_{1}\right), & k=1, \\
-V_{k-1} \cos \left(\phi_{k-1}-\phi_{k}\right)+2 V_{k} & \\
-V_{k+1} \cos \left(\phi_{k+1}-\phi_{k}\right), & k \neq k, N, \\
V_{N}-V_{N-1} \cos \left(\phi_{N-1}-\phi_{N}\right), & k=N,
\end{array}\right. \\
& \left(\omega-\frac{1}{\sqrt{L_{r} C_{k}}}\right) 4 R C_{k} \\
& = \begin{cases}\frac{\sin \left(\phi_{2}-\phi_{1}\right) V_{2},}{V_{1}}, & i=1, \\
+\frac{\sin \left(\phi_{k-1}-\phi_{k}\right) V_{k-1}}{V_{k}} & \\
-\frac{\sin \left(\phi_{k+1}-\phi_{k}\right) V_{k+1},}{V_{k}}, & k \neq k, N, \\
-\frac{\sin \left(\phi_{N-1}-\phi_{N}\right) V_{N-1}}{V_{N}}, & i=N .\end{cases}
\end{aligned}
$$

Using

$$
S_{k}= \begin{cases}1-\cos \left(\phi_{2}-\phi_{1}\right)\left(\frac{V_{2}}{V_{1}}\right), & k=1, \\
-\cos \left(\phi_{k-1}-\phi_{k}\right)\left(\frac{V_{k-1}}{V_{k}}\right)+2 & \\
-\cos \left(\phi_{k+1}-\phi_{k}\right)\left(\frac{V_{k+1}}{V_{k}}\right), & k \neq 1, N, \\
1-\cos \left(\phi_{N-1}-\phi_{N}\right)\left(\frac{V_{N-1}}{V_{N}}\right), & k=N, \\
U_{k}=\left\{\begin{array}{cc}
\sin \left(\phi_{2}-\phi_{1}\right)\left(\frac{V_{2}}{V_{1}}\right), & i=1, \\
+\sin \left(\phi_{k-1}-\phi_{k}\right)\left(\frac{V_{k-1}}{V_{k}}\right) & \\
-\sin \left(\phi_{k+1}-\phi_{k}\right)\left(\frac{V_{k+1}}{V_{k}}\right), & k \neq k, N, \\
-\sin \left(\phi_{N-1}-\phi_{N}\right)\left(\frac{V_{N-1}}{V_{N}}\right), & i=N,
\end{array}\right.\end{cases}
$$

the solution for the tuning parameters, $C_{t, k}\left(V_{k}^{t}\right)$ and $G_{t, k}\left(I_{k}^{t}\right)$, in terms of $V_{k}, \phi_{k}$ is

$$
\begin{gathered}
C_{k}=C_{o, k}\left(\frac{1}{2}\left[1 \pm \sqrt{1+\frac{U_{k}}{R} \sqrt{\frac{L_{r}}{C_{o, k}}}}\right]\right)^{2} \\
C_{t, k}=C_{k}-C_{r}
\end{gathered}
$$

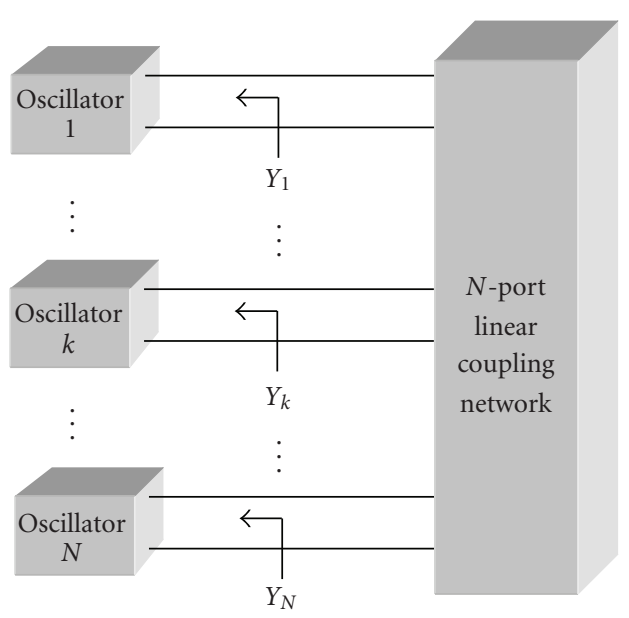

FIgURE 2: The coupled oscillator array.

$$
\begin{gathered}
V_{k}^{t}=V_{o}\left[\left(\frac{C_{o, k}}{\left(C_{k}-C_{r}\right)}\right)^{1 / \Gamma}-1\right], \\
G_{t, k}=\frac{-S_{k}}{2 R}-a_{k}+b_{k} V_{k}^{2}-G_{A}, \\
I_{k}^{t}=\frac{v_{o}}{G_{t, k}} .
\end{gathered}
$$

Before closing the section, the design procedure and check of the stability of the solution for the coupled oscillator array are listed. So, to design the mutually synchronized oscillator array in order to produce a given output voltage distribution (amplitudes and phases), the following steps are implemented.

(i) The given output amplitudes and phases, $V_{k}, \phi_{k}$, are inserted into (19) to produce the intermediate parameters $S_{k}$ and $U_{k}$.

(ii) In turn, $S_{k}$ and $U_{k}$ are inserted into (3). This results in computing the tuning parameters $C_{t, k}\left(V_{k}^{t}\right)$ and $G_{t, k}\left(I_{k}^{t}\right)$.

(iii) Inserting $G_{t, k}$ and $C_{t, k}$ into the differential equations (15) provides the evolution of the amplitudes and phases with time.

The key step of computing the proper $V_{k}, \phi_{k}$ is addressed next.

\section{The Synthesis Method}

The system of Figure 3 with the restriction of nonvarying interelement distance, $\left(\Delta z=z_{k}-z_{k-1}=\right.$ const $)$, has been used to control linear phased arrays in the limited cases of beam steering and pattern null in certain directions [8-13]. In the current study, we attempt a more general (not limited in certain cases) pattern synthesis. The system is designed by 


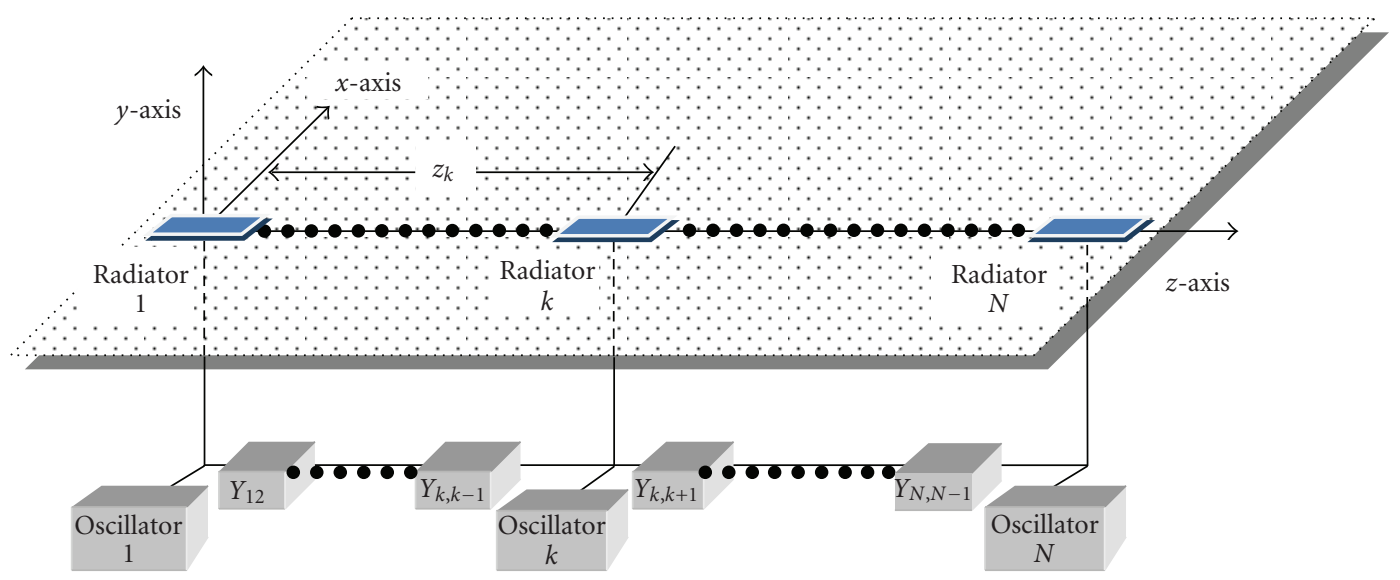

FIGURE 3: Bilateral injection-locking or mutual synchronization coupled oscillator's array.

providing the proper values for the positions of the radiators and for the voltage outputs of the oscillators. It is obvious that the proposed design offers specific advantages due to the degrees of freedom that make use.

To start the design of the array, we derive the cost function $C$ of the following form:

$$
C=\left\|F^{d}-F\right\|^{2}
$$

where

$$
\left\|F^{d}-F\right\|^{2}=\int_{0}^{\pi}\left|F^{d}(\theta)-F(\theta)\right|^{2} \sin (\theta) d \theta .
$$

We define

$$
A \circ B=\int_{\theta=0}^{\pi} A(\theta) \cdot B^{*}(\theta) \cdot \sin (\theta) d \theta
$$

From (23), (22) becomes

$$
\left\|F^{d}-F\right\|^{2}=\left(F^{d}-F\right) \circ\left(F^{d}-F\right)
$$

where $F^{d}(\theta)$ is the desired pattern, $F(\theta)$ is the resulting pattern, and $\left\|F^{d}-F\right\|^{2}$ is the mean square error. By taking into account the output voltage of the coupled oscillators, the resulting pattern can be expressed as

$$
F(\theta)=\sum_{k=1}^{N} \underbrace{\left[V_{k} e^{j \phi_{k}}\right]} F_{k},
$$

where $F_{k}=f_{k}(\theta) e^{j(2 \pi / \lambda) z_{k} \cos (\theta)}$.

We suppose a perturbation of the excitation of the oscillator outputs and of the position of the elements of the array. Due to the perturbation of the excitation, the new output of the $k$ th oscillator becomes $V_{k}+\Delta V_{k} ; \phi_{k}+\Delta \phi_{k}$. Also the new position of the $k$ th element is $z_{k}+\Delta z_{k}$. The pattern of the array results in

$$
\begin{aligned}
F(\theta)= & F_{0}(\theta)+\delta F(\theta) \\
\approx & \sum_{k=1}^{N} F_{k} V_{k} e^{j \phi_{k}}+\sum_{k=1}^{N} F_{k} e^{j \phi_{k}} \cdot \Delta V_{k} \\
& +j \sum_{k=1}^{N} F_{k} V_{k} e^{j \phi_{k}} \cdot \Delta \phi_{k}+\sum_{k=1}^{N} \frac{\partial F_{k}}{\partial z_{k}} \cdot V_{k} e^{j \phi_{k}} \cdot \Delta z_{k},
\end{aligned}
$$

where $F_{0}(\theta)$ is the initial pattern of the array.

It is shown in the Appendix that after assembling the perturbed values of the output voltage, $\left(\Delta V_{k}, \Delta \phi_{k}\right)$, of the oscillators and the position, $\left(\Delta z_{k}\right)$, of the elements in an unknown vector $[\Delta]$, the solution of the optimization problem leads to

$$
[\Delta]=[B]^{-1}[A]
$$

where $[A]$ and $[B]$ are proper matrices that include information relative to the specific problem. Expression (27) gives the appropriate perturbed values of the output voltage of the oscillators and the position of the elements. It is supposed that the desired pattern is not much different than the resulting one. In this case, the perturbation will produce the necessary changes in the excitation and the position of the elements. If $\left\|F^{d}-F\right\|^{2}$ is large, then the perturbation technique is applied iteratively until the mean square error becomes less than a given threshold. For further details on the functionality and the problems relative to the convergence of the proposed iterative method, the reader is referred to [5].

\section{Design Examples}

In the design examples that follow, the array can be derived by varying the excitation (amplitude and phase) simultaneously with the element positions. The desired far-field 
TABle 1: Van der Pol parameters of the employed oscillators.

\begin{tabular}{lccc}
\hline Osc. \# & I $(\sim 1 \mathrm{GHz})$ & II $(\sim 4.4 \mathrm{GHz})$ & III $(\sim 10 \mathrm{GHz})$ \\
Reference & {$[18]$} & {$[26]$} & {$[15]$} \\
\hline$a$ & 20.9657 & 0.0232 & 0.0036 \\
$b$ & 34.8669 & 0.0382 & 0.01 \\
$L_{r}$ & $0.214 \mathrm{pH}$ & $0.24 \mathrm{nH}$ & $10 \mathrm{pH}$ \\
$C_{r}$ & $105 \mathrm{nF}$ & $5.45 \mathrm{pF}$ & $23 \mathrm{pF}$ \\
$R_{A}=1 / G_{A}$ & $50 \Omega$ & $58 \Omega$ & $32 \Omega$ \\
\hline
\end{tabular}

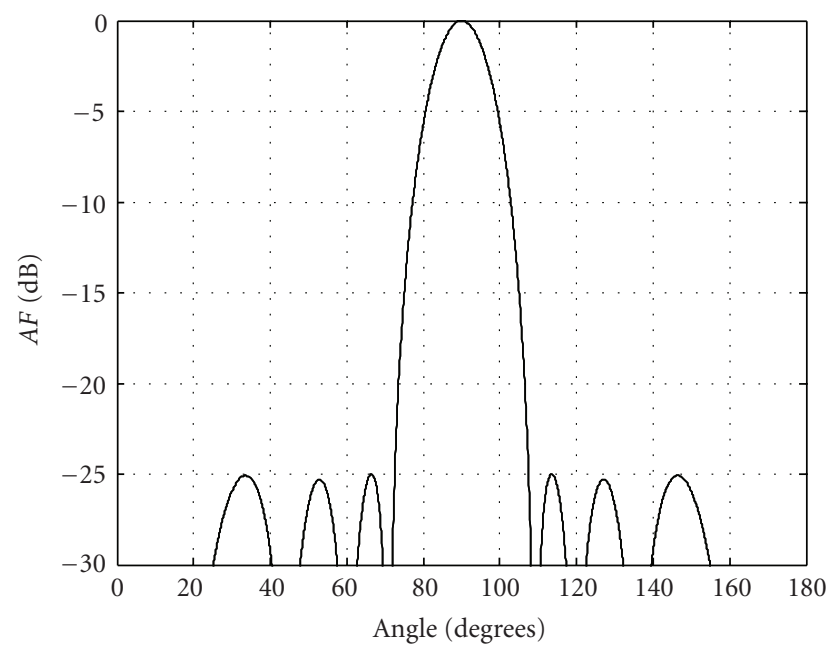

FIGURE 4: The attained pattern for the general design case.

pattern is a Chebyshev one with HPBW $=15$ degrees and SLL $=-25 \mathrm{~dB}$. We start from an array of 9 elements with uniform initial excitation and $0.45 \lambda$ interelement distance. We apply the synthesis method for three different cases. In case A, the excitations and the positions are to be derived. In case $B$, the phase of the excitations is set equal to zero, and in case $C$, the amplitude of the excitation is equal to unit.

For each one of the cases A, B, C, three different oscillators are employed to produce the respective far-field. The Van der Pol parameters of those oscillators are taken from published data (see Table 1).

The parameters of the coupling network are the same for all the examples as follows:

$$
Z_{o}=R=270 \Omega
$$

5.1. General Design Example. In this first example, both the element placement and the excitation are allowed to vary. After 20 iterations, the designed antenna array produces a pattern that is given in Figure 4.

The respective element placement and the excitations are given in Table 2.

The tuning parameters, $V_{k}^{t}$ and $I_{k}^{t}$, of the oscillators are computed after inserting the last two columns of Table 2 into (19)-(3). For the present case, the relative results are presented in Table 3.
TABle 2: Details on the general design case.

\begin{tabular}{llll}
\hline $\begin{array}{l}\text { Element } \\
\text { number }\end{array}$ & $\begin{array}{l}\text { Element } \\
\text { position } \\
(\lambda): z_{k}\end{array}$ & $\begin{array}{l}\text { Relative } \\
\text { excitation } \\
\text { amplitude: } \\
V_{k}\end{array}$ & $\begin{array}{l}\text { Excitation } \\
\text { phase } \\
\text { (degrees): } \\
\phi_{k}\end{array}$ \\
\hline 1 & -1.78 & 0.534 & 0.034 \\
2 & -1.17 & 0.842 & -0.806 \\
3 & -0.73 & 0.628 & -0.515 \\
4 & -0.36 & 1 & 0.516 \\
5 & 0 & 0.624 & 0.724 \\
6 & 0.36 & 1 & 0.516 \\
7 & 0.73 & 0.628 & -0.515 \\
8 & 1.17 & 0.842 & -0.806 \\
9 & 1.78 & 0.534 & 0.034 \\
\hline
\end{tabular}

The evolutions of $V_{k}(t)$ and $\phi_{k}(t)$ versus $t$ are produced after inserting into (15):

(1) the parameters of Table 1 and (28),

(2) the tuning parameter values, $C_{t, k}\left(V_{k}^{t}\right)$ and $G_{t, k}\left(I_{k}^{t}\right)$, and

(3) the initial, at $t=0$, amplitudes and phases:

$V_{k}(t=0)=$ random number uniformly distributed into $10^{-2}+(0,1) \cdot 10^{-3} \mathrm{~V}$,

$\phi_{k}(t=0)=$ random number uniformly distributed into $(0,2 \pi) \cdot 10^{-3}$.

In Figure 5, it is demonstrated that the designed coupled oscillator array can produce the necessary solution (see Table 2 and Figure 4) and furthermore, this solution is stable. In Figure 5(a), the voltage amplitudes of the nine oscillators versus time are given. Also, in Figure 5(b), the time evolution of the successive phase differences (output voltage phase of the $k$ th oscillator minus the output oscillator phase of the $(k-1)$ th oscillator) are given. It is clear that the amplitudes and phases converge to the correct values (continuous horizontal lines).

\subsection{Placement and Amplitude Design Example-Constant} Phases. From Table 2, it is shown that the phase of the elements is close to zero. Also, the final array is nonuniform in the excitation and the geometry. Namely, our solution is more complicated than the classical one for Chebyshev patterns $[1,2]$. Since we try to have a technically simple feeding network, we will solve the same problem with no phase difference between the elements and with only three different excitations. In Table 4, the final placement and the excitation of the elements, after 20 iterations, are given.

Also in Figure 6, the pattern of the array is presented. In the pattern, a difference of less than $0.5 \mathrm{~dB}$ between the desired and the resulting one is observed.

Comparing Table 2 with Table 4 , one can see that the second solution gives simpler results.

For the present case, the tuning parameters are given in Table 5. 
TABLE 3: The tuning parameters for the general design case.

\begin{tabular}{lcccccc}
\hline \multirow{2}{*}{ Element number } & \multicolumn{2}{c}{ OSC. I $(1.06 \mathrm{GHz})$} & \multicolumn{2}{c}{ OSC. II $(4.21 \mathrm{GHz})$} & \multicolumn{2}{c}{ OSC. III $(10.38 \mathrm{GHz})$} \\
& $V_{k}^{t}(\mathrm{mV})$ & $I_{k}^{t}(\mathrm{~mA})$ & $V_{k}^{t}(\mathrm{mV})$ & $I_{k}^{t}(\mathrm{~mA})$ & $V_{k}^{t}(\mathrm{mV})$ & $I_{k}^{t}(\mathrm{~mA})$ \\
\hline 1,9 & 1231.20 & 314 & 1207.79 & 0.440 & 1203.15 & 0.200 \\
2,8 & 1182.68 & 1402 & 1195.61 & 2.216 & 1198.22 & 1.455 \\
3,7 & 1171.23 & 593 & 1192.68 & 0.920 & 1197.03 & 0.547 \\
4,6 & 1212.10 & 2148 & 1203.04 & 3.460 & 1201.23 & 2.342 \\
5 & 1215.63 & 581 & 1203.92 & 0.910 & 1201.59 & 0.542 \\
\hline
\end{tabular}

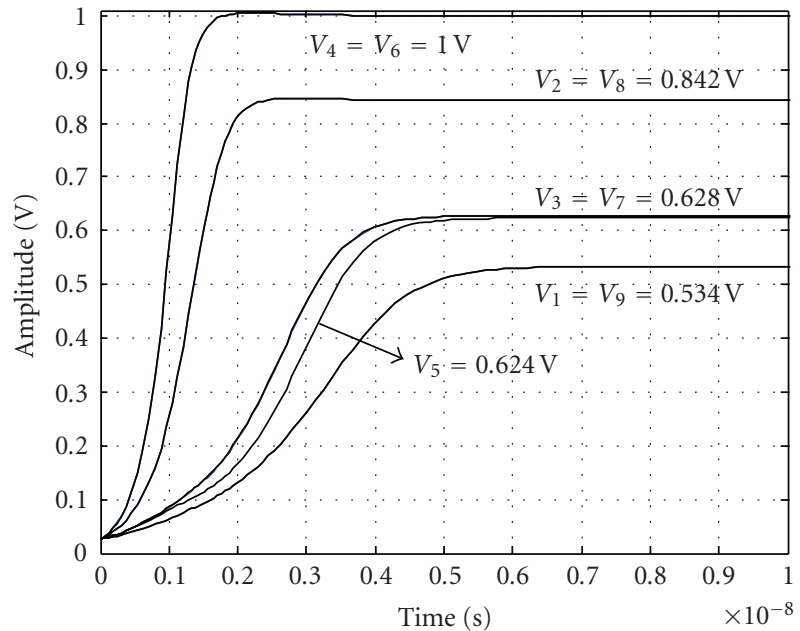

(a)

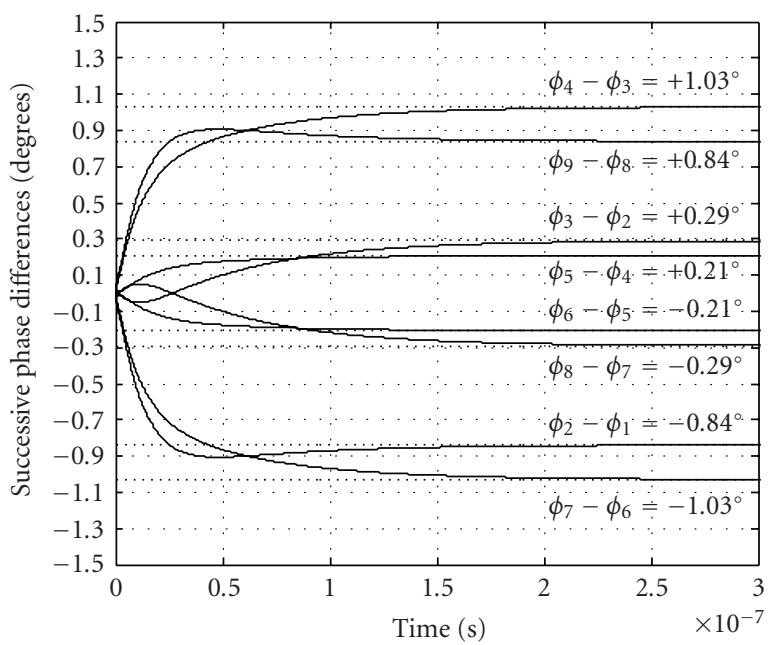

(b)

Figure 5: The coupled oscillator array response evolution with time for the general design case. (a) The evolution of the amplitudes. (b) The evolution of the phases.

In Figure 7, the time evolution curves for the amplitudes show the stability of the solution.

Note that comparing Figure 7 with Figure 5(a), different converging times are observed. In general, this fact is to be expected since the converging times, apart from the initial conditions $V_{k}(t=0), \phi_{k}(t=0)$, depend on the
Table 4: Details on the constant phase design case.

\begin{tabular}{llll}
\hline $\begin{array}{l}\text { Element } \\
\text { number }\end{array}$ & $\begin{array}{l}\text { Element } \\
\text { position } \\
(\lambda): z_{k}\end{array}$ & $\begin{array}{l}\text { Relative } \\
\text { excitation } \\
\text { amplitude: } \\
V_{k}\end{array}$ & $\begin{array}{l}\text { Excitation } \\
\text { phase } \\
\text { (degrees): } \\
\phi_{k}\end{array}$ \\
\hline 1 & -1.779 & 0.4 & 0 \\
2 & -1.386 & 0.4 & 0 \\
3 & -0.961 & 0.8 & 0 \\
4 & -0.464 & 1 & 0 \\
5 & 0 & 1 & 0 \\
6 & 0.464 & 1 & 0 \\
7 & 0.961 & 0.8 & 0 \\
8 & 1.386 & 0.4 & 0 \\
9 & 1.779 & 0.4 & 0 \\
\hline
\end{tabular}

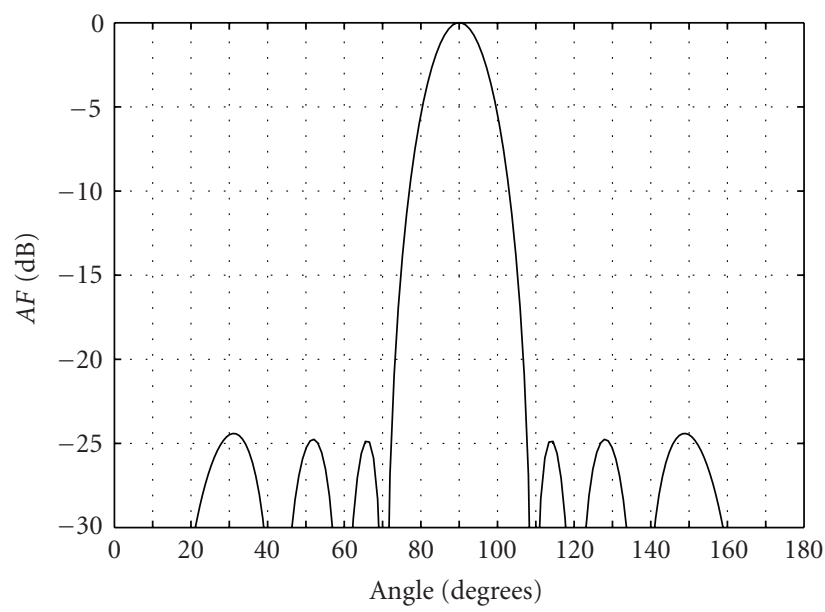

FIgURE 6: The attained pattern for the constant phase design case.

tuning parameter values that are different for the two design examples.

5.3. Placement and Phase Design Example-Constant Amplitudes. In this last case, we provide a design where the amplitudes are equal. The attained solution is given in Table 6.

The produced far-field pattern is given in Figure 8.

It is noted that in this case, the SLL criterion of $-25 \mathrm{~dB}$ is not fulfilled, (SLL $=-21.5 \mathrm{~dB}$ ). The above shows that the phases of the elements are not enough to solve the problem. 
TABLE 5: The tuning parameters for the constant phase design case.

\begin{tabular}{|c|c|c|c|c|c|c|}
\hline \multirow{2}{*}{ Element number } & \multicolumn{2}{|c|}{ OSC. I (1.06 GHz) } & \multicolumn{2}{|c|}{ OSC. II $(4.21 \mathrm{GHz})$} & \multicolumn{2}{|c|}{ OSC. III (10.38 GHz) } \\
\hline & $V_{k}^{t}(\mathrm{mV})$ & $I_{k}^{t}(\mathrm{~mA})$ & $V_{k}^{t}(\mathrm{mV})$ & $I_{k}^{t}(\mathrm{~mA})$ & $V_{k}^{t}(\mathrm{mV})$ & $I_{k}^{t}(\mathrm{~mA})$ \\
\hline 1,9 & 1200 & 314.1 & 1200 & 0.445 & 1200 & 0.203 \\
\hline 2,8 & 1200 & 313.4 & 1200 & 0.483 & 1200 & 0.240 \\
\hline 3,7 & 1200 & 2515 & 1200 & 4.196 & 1200 & 2.892 \\
\hline 4,6 & 1200 & 4166 & 1200 & 7.019 & 1200 & 4.918 \\
\hline 5 & 1200 & 4166 & 1200 & 7.028 & 1200 & 4.924 \\
\hline
\end{tabular}

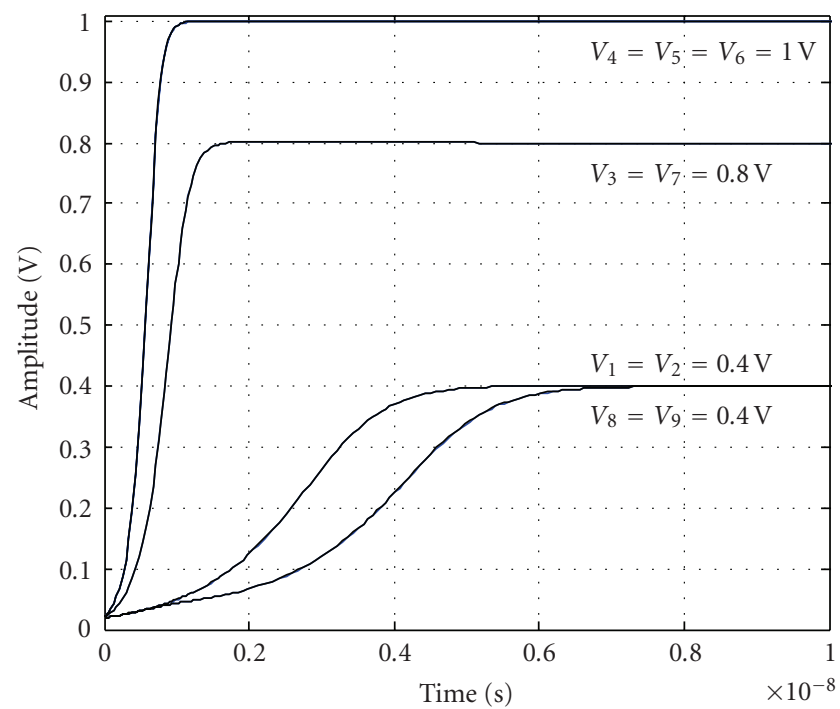

FIGURE 7: The coupled oscillator array response evolution with time for the constant phase design case. The evolution of the amplitudes.

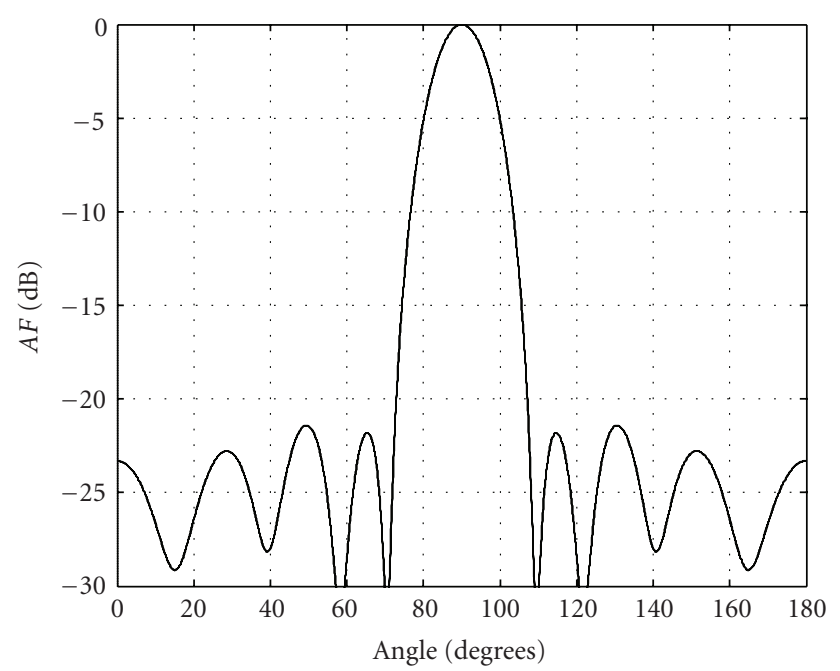

FIGURE 8: The attained pattern for the constant amplitude design case.

For the present case, the tuning parameters are given in Table 7 .

For the resulting pattern, the respective evolution curves are given in Figure 9.
TABLE 6: Details on the constant amplitude design case.

\begin{tabular}{llll}
\hline $\begin{array}{l}\text { Element } \\
\text { number }\end{array}$ & $\begin{array}{l}\text { Element } \\
\text { position } \\
(\lambda): z_{k}\end{array}$ & $\begin{array}{l}\text { Relative } \\
\text { excitation } \\
\text { amplitude: } \\
V_{k}\end{array}$ & $\begin{array}{l}\text { Excitation } \\
\text { phase } \\
\text { (degrees): } \\
\phi_{k}\end{array}$ \\
\hline 1 & -1.59 & 1 & -0.043 \\
2 & -0.98 & 1 & -4.121 \\
3 & -0.70 & 1 & +4.909 \\
4 & -0.26 & 1 & -6.975 \\
5 & 0 & 1 & -5.936 \\
6 & 0.26 & 1 & -6.975 \\
7 & 0.70 & 1 & +4.909 \\
8 & 0.98 & 1 & -4.121 \\
9 & 1.59 & 1 & -0.043 \\
\hline
\end{tabular}

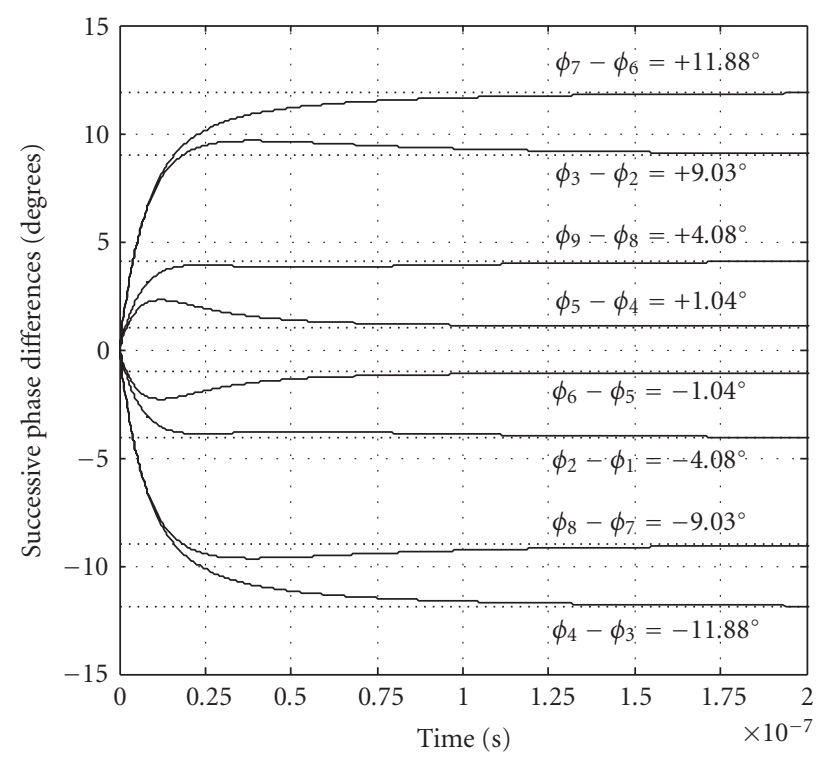

FIGURE 9: The coupled oscillator array response evolution with time for the constant amplitude design case. The evolution of the phases.

It is obvious that in the array synthesis, one must tradeoff the simplicity of networks, the size of the array, and the number and type of the different excitations. From the presented cases, it is assessed that the general one is rather complex, while the constant amplitude case fails to comply 
TABLE 7: The tuning parameters for the constant amplitude design case.

\begin{tabular}{lccccc}
\hline \multirow{2}{*}{ Element number } & \multicolumn{2}{c}{ OSC. I $(1.06 \mathrm{GHz})$} & \multicolumn{2}{c}{ OSC. II $(4.21 \mathrm{GHz})$} & \multicolumn{2}{c}{ OSC. III (10.38 GHz) } \\
& $V_{k}^{t}(\mathrm{mV})$ & $I_{k}^{t}(\mathrm{~mA})$ & $V_{k}^{t}(\mathrm{mV})$ & $I_{k}^{t}(\mathrm{~mA})$ & $V_{k}^{t}(\mathrm{mV})$ \\
\hline 1,9 & 1298.56 & 314.0 & 1224.13 & 0.401 & 1209.73 \\
2,8 & 930.27 & 314.0 & 1125.81 & 0.401 & 1169.32 \\
3,7 & 1805.51 & 314.0 & 1328.56 & 0.400 & 1250.49 \\
4,6 & 934.48 & 314.0 & 1127.07 & 0.400 & 1169.85 \\
5 & 1249.32 & 314.0 & 1212.25 & 0.401 & 1204.95 \\
\hline
\end{tabular}

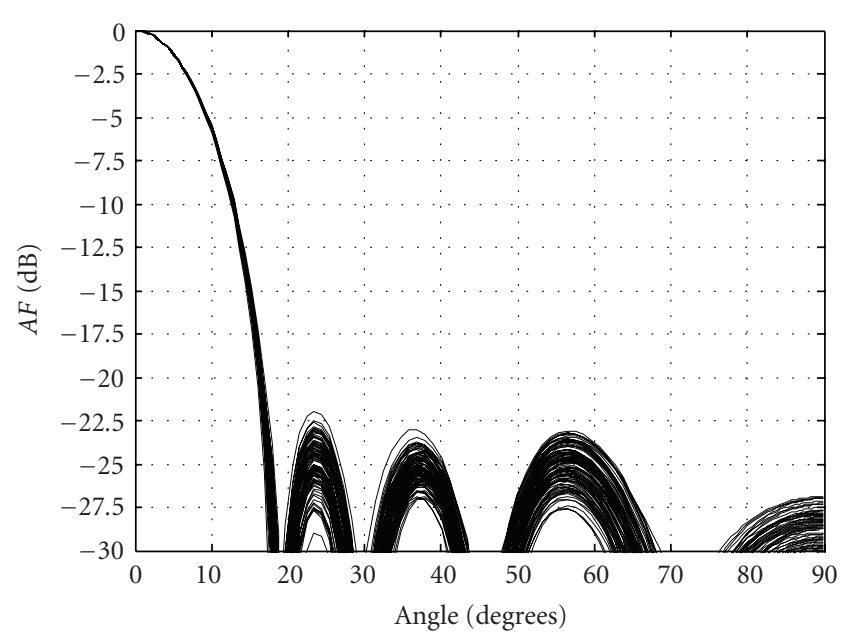

Figure 10: The attained amplitude sensitivity pattern for the general design case.

with the desired pattern. It seems that the constant phase case presents a better compromise between complexity and performance of the device under study.

Before closing the section, to evaluate the validity of the presented method and the viability of the listed results, some comments are in order. Both phase and amplitude control should be implemented with a relatively easy way. Varactor and PIN diodes are simple electronic elements that should be used. That is because varactor diodes are extensively used as tuning elements in conventional VCOs, and also PIN diodes are employed as variable resistors for vector modulator circuits. The issue that raises concern is the imprecise setting of the values of the tuning parameters and the effect that manufacturing tolerances will have on the overall performance of the system. Fabrication and tuning inconsistencies among individual oscillators in an array lead to differences in the free-running frequencies and amplitudes. This randomness causes phase and amplitude errors that are expected to result in pattern deformation manifested in beam-pointing and SLL errors $[1,30]$.

To provide understanding of the relationships among random tuning error distribution and pattern deformation, a statistical study, focused on the sensitivity analysis, is provided through Monte Carlo simulation. The element placement, the tuning voltage, and the tuning current are treated as random vectors following normal probability distribution with means given by the previous analysis and varying values of variance. In each statistical experiment, after choosing the placement and tuning parameters, $C_{t, k}\left(V_{k}^{t}\right)$ and $G_{t, k}\left(I_{k}^{t}\right)$ are used to extract the time evolution of $V_{k}(t)$ and $\phi_{k}(t)$. At the steady state, the far field of the array is computed. The results show that when only $I_{k}^{t}$ is varied and if two or three dBs degradation on SLL performance are acceptable, then the variance should be no more than $10 \%$ of the parameters nominal value. This is shown in Figure 10 when all the patterns resulted from a 100-run relative Monte Carlo experiment are depicted. Nearly, for one and two orders of magnitude, less variation is needed for attaining the same results when position and phase are varied, respectively. This means that position and amplitudes setting problems are expected to be less harsh compared to the problems that have already encountered in setting the phase [31], in conventional VCOs. If the previously-stated performance degradation is unacceptable, properly chosen calibration procedure should be considered $[32,33]$.

\section{Conclusion}

A design procedure for the synthesis of active antenna arrays is presented. Those arrays are sets of radiator oscillator couples with each element connecting through a coupling network to the others. Such a system presents the designer with two sets of parameters: the radiators' positions and the oscillators' outputs, the second used to excite the first.

The procedure designs simultaneously both the antenna and the attached coupled oscillator array providing viable solutions. In this way, the current work demonstrates how to control the dynamics of coupled oscillator arrays in order to achieve pattern shaping. Whereas previous work has been almost exclusively devoted to beam steering and null formation, this technique extends the versatility of coupled oscillator arrays through manipulation of the aperture amplitude and phase distribution. Furthermore, simultaneously an amplitude space taper distribution, $(\Delta z \neq 0)$, is attained to produce the desired far-field pattern.

The implementability of our method is based first on the actually monitoring of the time evolution of the oscillators' outputs (27). Afterward, the existence of the proper varactors and PINs by using (3) is addressed. It is obvious that the limited range of their values should set some constraints on the resultant pattern. So, in some cases, a proper iterative combination between the capacitance and conductance and the desired pattern is important. 
For all design cases, and independently on the complexity of the specific problem, the required excitation distribution can be readily implemented with proper tune of control parameters, such as the varactor capacitance and the PIN conductance of the oscillators. The relative closed-form formulas are presented for the case of the mutually synchronized coupled oscillator array.

\section{Appendix}

Following (25) and (26), the matrix form the initial pattern and its first variation can be written as

$$
\begin{aligned}
& F_{0}(\theta) \\
& =[F]^{T}[\tilde{V}]=[\tilde{V}]^{T}[F], \\
& \delta F(\theta) \\
& =\operatorname{diag}\left(\frac{[\tilde{V}]}{[V]}\right)[F]^{T}[\Delta V] \\
& \quad+j \operatorname{diag}([\tilde{V}])[F]^{T}[\Delta \phi]+\operatorname{diag}([\tilde{V}])[\dot{F}]^{T}[\Delta z] \\
& =\left[\operatorname{diag}\left(\frac{[\tilde{V}]}{[V]}\right)[F]^{T} \quad j \operatorname{diag}([\tilde{V}])[F]^{T} \operatorname{diag}([\tilde{V}])[\dot{F}]^{T}\right] \\
& \quad \times\left[\begin{array}{c}
{[\Delta V]} \\
{[\Delta \phi]} \\
{[\Delta z]}
\end{array}\right],
\end{aligned}
$$

where

$$
\begin{gathered}
{[F]=\left[\begin{array}{lllll}
F_{1} & \cdots & F_{k} & \cdots & F_{N}
\end{array}\right]^{T},} \\
{[\tilde{V}]=\left[\begin{array}{lllll}
V_{1} e^{j \phi_{1}} & \cdots & V_{k} e^{j \phi_{k}} & \cdots & V_{N} e^{j \phi_{N}}
\end{array}\right]^{T},} \\
{[1]=\left[\begin{array}{lllll}
1 & \cdots & 1 & \cdots & 1
\end{array}\right]^{T},} \\
{[\Delta V]=\left[\begin{array}{lllll}
\Delta V_{1} & \cdots & \Delta V_{k} & \cdots & \Delta V_{N}
\end{array}\right]^{T},} \\
{[\Delta \phi]=\left[\begin{array}{lllll}
\Delta \phi_{1} & \cdots & \Delta \phi_{k} & \cdots & \Delta \phi_{N}
\end{array}\right]^{T},} \\
{[\Delta z]=\left[\begin{array}{lllll}
\Delta z_{1} & \cdots & \Delta z_{k} & \cdots & \Delta z_{N}
\end{array}\right]^{T},}
\end{gathered}
$$

where $[\cdot]^{T}$ stands for the transpose operation. Following (A.1) and (A.2), the new pattern is written as

$$
\begin{aligned}
F(\theta)= & F_{0}(\theta)+\delta F(\theta) \\
= & \operatorname{diag}([\tilde{V}])[F]^{T}[1]+\operatorname{diag}\left(\frac{[\tilde{V}]}{[V]}\right)[F]^{T}[\Delta V] \\
& +j \operatorname{diag}([\tilde{V}])[F]^{T}[\Delta \phi]+\operatorname{diag}([\tilde{V}])[\dot{F}]^{T}[\Delta z] .
\end{aligned}
$$

Inserting (A.3) into (24), after defining a vector $\Delta$ as

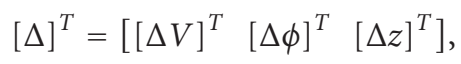

we have

$$
\begin{aligned}
\left\|F^{d}-F\right\|^{2}= & \left\|F^{d}-F_{0}\right\|^{2}-[\Delta]^{T}[A]-[A]^{T}[\Delta] \\
& +[\Delta]^{T}[B][\Delta] .
\end{aligned}
$$

In (A.5), it is

$$
A=\left(\left[\begin{array}{c}
\operatorname{diag}\left(\frac{[\tilde{V}]}{[V]}\right)[F] \\
j \operatorname{diag}([\tilde{V}])[F] \\
\operatorname{diag}([\tilde{V}])[\dot{F}]
\end{array}\right] \circ\left(F^{d}-F_{0}\right)\right),
$$

$$
B=\left(\left[\begin{array}{c}
\operatorname{diag}\left(\frac{[\tilde{V}]}{[V]}\right)[F] \\
j \operatorname{diag}([\tilde{V}])[F] \\
\operatorname{diag}([\tilde{V}])[\dot{F}]
\end{array}\right] \circ\left[\begin{array}{c}
\operatorname{diag}\left(\frac{[\tilde{V}]}{[V]}\right)[F] \\
j \operatorname{diag}([\tilde{V}])[F] \\
\operatorname{diag}([\tilde{V}])[\dot{F}]
\end{array}\right]^{T}\right) .
$$

By zeroing the first variation of $\left\|F^{d}-F\right\|^{2}$ with respect the $[\Delta]^{T}$, we have

$$
\frac{\delta\left\|F^{d}-F\right\|^{2}}{\delta[\Delta]^{T}}=-[A]+[B][\Delta]=0 .
$$

The above gives the vector $[\Delta]$ :

$$
[\Delta]=[B]^{-1}[A] .
$$

\section{References}

[1] R. C. Hansen, Phased Array Antennas, John Wiley \& Sons, New York, NY, USA, 1998.

[2] C. L. Dolph, "A current distribution for broadside arrays which optimizes the relationship between beamwidth and sidelobe level," Proceedings of the IRE, vol. 34, no. 6, pp. 335348,1946

[3] H. J. Orchard, R. S. Elliott, and G. J. Stern, "Optimising the synthesis of shaped beam antenna patterns," IEE Proceedings H: Microwaves, Antennas and Propagation, vol. 132, no. 1, pp. 63-68, 1985.

[4] J. N. Sahalos, Orthogonal Methods for Array Synthesis: Theory and the ORAMA Computer Tool, John Wiley \& Sons, West Sussex, UK, 2006.

[5] T. N. Kaifas and J. N. Sahalos, "On the geometry synthesis of arrays with a given excitation by the orthogonal method," IEEE Transactions on Antennas and Propagation, vol. 56, no. 12, pp. 3680-3688, 2008.

[6] R. Mailloux, Phased Array Antenna Handbook, Artech House, Boston, Mass, USA, 2nd edition, 2005.

[7] K. Chang, R. A. York, P. S. Hall, and T. Itoh, "Active integrated antennas," IEEE Transactions on Microwave Theory and Techniques, vol. 50, no. 3, pp. 937-944, 2002.

[8] K. D. Stephan and W. A. Morgan, "Analysis of inter-injectionlocked oscillators for integrated phased arrays," IEEE Transactions on Antennas and Propagation, vol. 35, no. 7, pp. 771-781, 1987.

[9] P. Liao and R. A. York, "A new phase-shifterless beamscanning technique using arrays of coupled oscillators," IEEE Transactions on Microwave Theory and Techniques, vol. 41, no. 10, pp. 1810-1815, 1991.

[10] R. J. Pogorzelski, R. P. Scaramastra, J. Huang, R. J. Beckon, S. M. Petree, and C. M. Chavez, "A seven-element S-band coupled-oscillator controlled agile-beam phased array," IEEE Transactions on Microwave Theory and Techniques, vol. 48, no. 8, pp. 1375-1384, 2000. 
[11] T. Heath, "Simultaneous beam steering and null formation with coupled, nonlinear oscillator arrays," IEEE Transactions on Antennas and Propagation, vol. 53, no. 6, pp. 2031-2035, 2005.

[12] A. Georgiadis, A. Collado, and A. Suarez, "Pattern nulling in coupled oscillator antenna arrays," IEEE Transactions on Antennas and Propagation, vol. 55, no. 5, pp. 1267-1274, 2007.

[13] T. Heath, "Beam steering of nonlinear oscillator arrays through manipulation of coupling phases," IEEE Transactions on Antennas and Propagation, vol. 52, no. 7, pp. 1833-1842, 2004.

[14] K. Kurokawa, "Injection locking of solid-state microwave oscillators," Proceedings of the IEEE, vol. 61, no. 10, pp. 13861410, 1973.

[15] K. D. Stephan, "Inter-injection-locked oscillators for power combining and phased arrays," IEEE Transactions on Microwave Theory Techniques, vol. 34, no. 10, pp. 10171025, 1986.

[16] K. Y. Chen, P. D. Biernacki, A. Lahrichi, and A. Mickelson, "Analysis of an experimental technique for determine Van der Pol parameters of a transistor oscillator," IEEE Transactions on Microwave Theory Techniques, vol. 46, no. 7, pp. 914-922, 1998.

[17] S. Yang, V. F. Fusco, and D. E. J. Humphrey, "Ring-coupledoscillator sequentially rotated active antenna," IEEE Transactions on Microwave Theory and Techniques, vol. 49, no. 8, pp. 1492-1497, 2001.

[18] S. Drew and V. F. Fusco, "A software tool for active antenna array dynamical evaluation," International Journal of Numerical Modelling: Electronic Networks, Devices and Fields, vol. 10, no. 3, pp. 169-176, 1997.

[19] N. H. W. Fong, J.-O. Plouchart, N. Zamdmer, et al., "A 1-V 3.8-5.7-GHz wide-band VCO with differentially tuned accumulation MOS varactors for common-mode noise rejection in CMOS SOI technology," IEEE Transactions on Microwave Theory and Techniques, vol. 51, no. 8, pp. 1952-1959, 2003.

[20] P. Andreani and S. Mattisson, "On the use of MOS varactors in RF VCO's," IEEE Journal of Solid-State Circuits, vol. 35, no. 6, pp. 905-910, 2000.

[21] R. Mukhopadhyay, Y. Park, P. Sen, et al., "Reconfigurable RFICs in Si-based technologies for a compact intelligent RF front-end," IEEE Transactions on Microwave Theory and Techniques, vol. 53, no. 1, pp. 81-93, 2005.

[22] Microsemi Corporation, The PIN Diode Circuit Designer's Handbook, Microsemi, Lowell, Mass, USA, 1998.

[23] U. L. Rohde and D. P. Newkirk, RF/Microwave Circuit Design for Wireless Applications, John Wiley \& Sons, New York, NY, USA, 2000.

[24] A. H. E. Ashtiani, S.-I. Nam, A. d'Espona, S. Lucyszyn, and L. D. Robertson, "Direct multilevel carrier modulation using millimeter-wave balanced vector modulators," IEEE Transactions on Microwave Theory and Techniques, vol. 46, no. 12, part 2, pp. 2611-2619, 1998.

[25] R. A. York, P. Liao, and J. J. Lynch, "Oscillator array dynamics with broadband N-port coupling networks," IEEE Transactions on Microwave Theory and Techniques, vol. 42, no. 11, pp. 2040-2045, 1994.

[26] R. A. York, "Nonlinear analysis of phase relationships in quasioptical oscillator arrays," IEEE Transactions on Microwave Theory and Techniques, vol. 41, no. 10, pp. 1799-1809, 1993.

[27] D. E. J. Humphrey and V. F. Fusco, "Active antenna array lumped ring configuration," IEEE Transactions on Antennas and Propagation, vol. 46, no. 9, pp. 1279-1284, 1998.
[28] R. A. York and T. Itoh, "Injection- and phase-locking techniques for beam control," IEEE Transactions on Microwave Theory and Techniques, vol. 46, no. 11, part 2, pp. 1920-1929, 1998.

[29] R. J. Pogorzelski, "On the design of coupling networks for coupled oscillator arrays," IEEE Transactions on Antennas and Propagation, vol. 51, no. 4, pp. 794-801, 2003.

[30] J. Litva and T. Lo, Beamforming in Wireless Communication, Artech House, Boston, Mass, USA, 1996.

[31] J. Shen and L. W. Pearson, "The phase error and beampointing error in coupled oscillator beam-steering arrays," IEEE Transactions on Antennas and Propagation, vol. 53, no. 1, part 2, pp. 386-393, 2005.

[32] C. T. Charles, A calibrated phase and amplitude control system for phased-array transmitters, Ph.D. thesis, University of Washington, Seattle, Wash, USA, 2006.

[33] R. J. Pogorzelski, "A 5-by-5 element coupled oscillator-based phased array," IEEE Transactions on Antennas and Propagation, vol. 53, no. 4, pp. 1337-1345, 2005. 

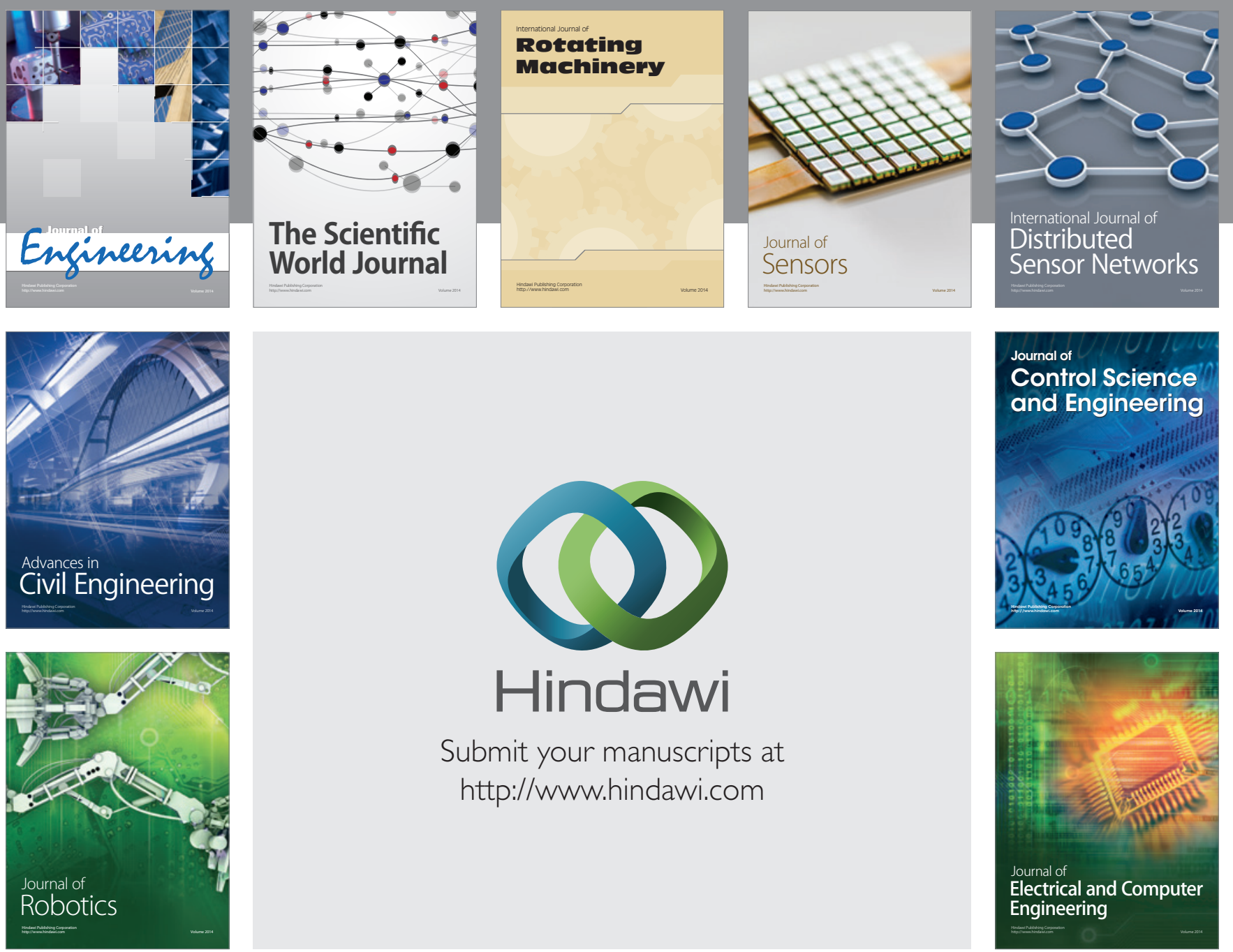

Submit your manuscripts at

http://www.hindawi.com
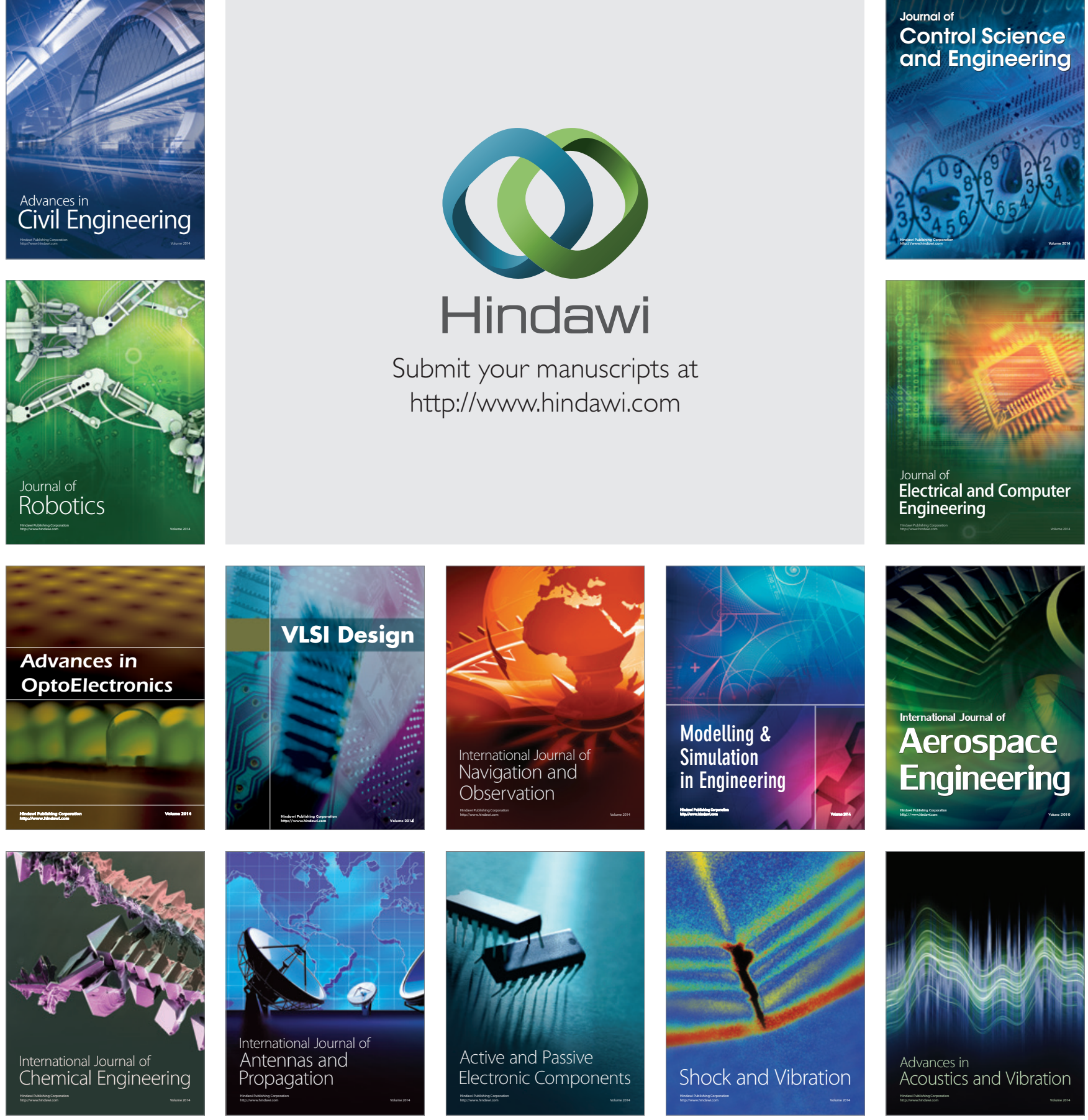